\title{
ZWISCHEN SPRACH- UND REZEPTIONSÖKONOMIE. ZU VERWEISUNGEN ALS MITTEL DER TEXTVERDICHTUNG AN BEISPIELEN AUS DEM DEUTSCHEN STGB
}

\author{
KAROLINA KĘSICKA, Dr. \\ Adam-Mickiewicz-Universität \\ Institut für Germanische Philologie \\ Poznań, Polen \\ kesicka@amu.edu.pl
}

\begin{abstract}
Im Zeitalter der globalisierten Wissensvermittlung und Kommunikation ist die Sprachökonomie zu einem relevanten Faktor im Gestaltungsprozess von Fachtexten geworden. Sie hat die Effizienz fachlicher Kommunikation und zugleich die Minimierung des Textumfangs $\mathrm{zu}$ gewährleisten. Zur Förderung der Kommunikation werden mehrere Mittel und Techniken der Textverknappung entwickelt, darunter Verweisungen, die ein häufig verwendetes Verdichtungsmittel in Gesetzestexten darstellen. Der vorliegende Beitrag setzt sich zum Ziel, verschiedene Verweisformen im Hinblick auf ihre Textfunktion und ihren Einfluss auf die Textrezeption zu untersuchen. An Beispielen aus dem deutschen StGB wird versucht $\mathrm{zu}$ erforschen, ob sich Verweisungen in der Tat immer als rezeptionsökonomisch erweisen. Ausgehend von theoretischen Reflexionen über das Phänomen der Sprachökonomie wollen wir anschließend zur empirischen Textanalyse vom Standpunkt des Lesers übergehen, um zu analysieren, worin die potentiellen sprachökonomisch bedingten Rezeptionsprobleme liegen können und wie sie die Textrezeption beeinflussen.
\end{abstract}


Schlüsselwörter: Sprachökonomie, Textverdichtung, Verweisungen, Textrezeption, Rechtstext

\title{
MIĘDZY EKONOMIĄ JĘZYKA A RECEPCJĄ TEKSTU. ODESŁANIA JAKO ŚRODEK SŁUŻĄCY KONDENSACJI TREŚCI NA PRZYKLADACH Z NIEMIECKIEGO KODEKSU KARNEGO
}

\begin{abstract}
Abstrakt:W dobie globalizacji wiedzy i komunikacji zjawisko ekonomii języka stało się ważną determinantą $\mathrm{w}$ procesie tworzenia tekstów fachowych. Ma ono zagwarantować efektywność komunikacji przy jednoczesnej minimalizacji środków językowych. By optymalizować proces komunikacji, rozwinięto szereg środków umożliwiających kondensację treści w tekście. Jednym z nich są odesłania służące osiąganiu zwięzłości w tekstach prawnych. Niniejszy artykuł ma na celu przedstawienie różnych form odesłań i analizę ich roli w procesie recepcji tekstu. $\mathrm{Na}$ wybranych przykładach z niemieckiego kodeksu karnego prześledzimy, czy zawsze rzeczywiście wspierają one rozumienie tekstu i jakie mogą być przyczyny ich nieefektywności z punktu widzenia ekonomiczności przekazu informacji.
\end{abstract}

Słowa kluczowe: ekonomia języka, kondensacja tekstu, odesłania, recepcja tekstu, tekst prawny

\section{BETWEEN LANGUAGE ECONOMY AND TEXT RECEPTION. ABOUT REFERENCES AS A METHOD OF TEXT CONDENSATION ON THE EXAMPLES FROM THE GERMAN CRIMINAL CODE}

\begin{abstract}
In the era of globalized knowledge and communication language economy has become important determining factor in the creation process of specialized texts. The language economy has to ensure effective communication and to reduce the cognitive effort required for a text reception and production. To optimize the communication process, a variety of measures have been developed to make text condensation possible. One of these measures are references in legal texts. The purpose of this paper is to present different types of references combined with the analysis of their text function and role in the process of text reception. By empirically examining of the selected examples from the German Criminal Code, we will examine whether the language economy always helps the reader to understand legal texts? Finally, our focus is on showing of the possible causes for the communication inefficiency of examined references.
\end{abstract}

Key words: language economy, text condensation, references, text reception, legal text 


\section{Vorüberlegungen}

Im Zeitalter der globalisierten Wissensvermittlung und Kommunikation wird die Sprachökonomie zu einer relevanten Determinante im Produktionsprozess von Fachtexten. Einerseits hat sie es zu gewährleisten, die Effektivität der Fachkommunikation zu steigern und andererseits soll sie den kognitiven Aufwand der Kommunikationspartner u.a. durch die Minderung des Textumfangs minimieren. Um den Kommunikationsprozess optimieren zu können, werden mehrere sprachökonomische Mittel und Techniken der Textverknappung sowie -verdeutlichung entwickelt, die auf die Prägnanz des Inhalts und die Entlastung der Kognitionsprozesse bei der Textrezeption abzielen.

Der vorliegende Beitrag setzt sich zum Ziel, Verweisungen als ein Verdichtungsmittel näher zu betrachten, das in Gesetzestexten besonders häufig angewendet wird. An Beispielen aus dem deutschen StGB soll analysiert werden, inwieweit verschiedene Verweisformendie Textqualität beeinflussen und die Rezeptionsökonomie fördern. Unser besonderes Augenmerk gilt dabei der Fragestellung, ob in der Tat die Sprachökonomie der Rezeptionsökonomie gleichkommt. Führt die Ausdrucksknappheit immer zur Erreichung der angestrebten kommunikativen Zielsetzung, und fördert sie somit das Textverstehen? Oder können sich sprachlich unökonomische Lösungen doch als rezeptionsökonomischer erweisen, obwohl sie einen größeren kognitiven Aufwand erforderlich machen?

Ausgehend von theoretischen Reflexionen über das Phänomen der Sprachökonomie wollen wir anschließend zur empirischen Textanalyse vom Standpunkt des Lesers übergehen, um eine Antwort auf die genannten Fragestellungen zu versuchen. 
Karolina Kęsicka: ZwischenSprach- und ...

\section{Sprachökonomie - Begriffsweite und Formenvarianz}

Auf das Spannungsverhältnis zwischen der Sprach- und Rezeptionsökonomie, das auf die einleitend genannte Widersprüchlichkeit von Bestrebungen zurückzuführen ist, wird bereits in den 1960er Jahren verwiesen, als Martinet auf die Antinomie zwischen den Kommunikationsbedürfnissen des Menschen und seinem Bestreben, geistige und körperliche Tätigkeiten auf ein Minimum zu reduzieren, hindeutet (vgl. Martinet 1960: 182). In der Alltags- und Fachkommunikation wird nach Klarheit, Deutlichkeit und Präzision gestrebt, „dieses Streben wirkt sich [allerdings] oft gegen die Neigung zur Bequemlichkeit, zu Kraftersparnis aus“ (Moser 1971: 89).

Wie Moser betont, erwächst das Phänomen der sprachlichen Ökonomie aus einem Komplex von gegenläufigen Bestrebungen (vgl. Moser 1971: 90). Jeder Mensch und jede Generation verlangt nach Neuerungen, weist eine natürliche Neigung zum Fortschritt auf und hat ein stetes Bedürfnis nach Kommunikation. Auf diese Anstöße ist letztendlich die menschliche, auch die sprachliche Entwicklung zurückzuführen. Der Mensch tendiert zu gleichaber auch dazu, „Neues mit möglichst wenig Kraftaufwand zu verwirklichen" (Moser 1971: 90). Darin mag eine Erklärung für das Bestreben gefunden werden, bereits bestehende sprachliche Grundmuster weiter zu entwickeln, damit sie sich besser an neue Kommunikationsbedürfnisse anpassen, anstatt das Sprachsystem jedes Mal neu zu bilden, wenn eine neue Kommunikationssituation entsteht. Diese Vorüberlegungen verleiten Moser zur Formulierung folgender Definition sprachlicher Ökonomie:

„Wir verstehen unter sprachlicher Ökonomie das Streben bewusster und unbewusster und teilbewusster Art,

1.1. sprachliche Mittel einzusparen und dadurch bei der sprachlichen Betätigung den physischen und geistigen Kraftaufwand $\mathrm{zu}$ verringern,

1.2. diesen auch beim Ausbau der sprachlichen Mittel möglichst klein zu halten,

2 die Leistungsfähigkeit, die Effizienz der sprachlichen Mittel zu erhöhen, 


\section{ComparativeLegilinguistics 29/2017}

3 die regionalen und sozialen Normverschiedenheiten auszugleichen und dadurch den Kommunikationsbedürfnissen besser gerecht zu werden.“ (Moser 1971: 92)

Aus der Mehrzahl von Gesichtspunkten, die bei der Erfassung des Phänomens mit einbezogen werden, ergeben sich für Moser grundsätzlich drei Typen sprachlicher Ökonomie: die systembezogene Ökonomie, die informationsbezogene Ökonomie und Geltungsökonomie, die sich auf die räumlich-soziale Vereinheitlichung des Sprachsystems bezieht (vgl. Moser 1971: 93). Für die Untersuchungszwecke des vorliegenden Beitrags erweisen sich die ersten zwei als besonders relevant, deswegen wird der Typus IIIaußer Acht gelassen.

\subsection{Systembezogene Ökonomie}

Unter die systembezogene Ökonomie subsumiert Moser mehrere Erscheinungen, denen das Streben nach

(i) der Reduzierung des physischen und kognitiven Aufwands beim Sprachgebrauch,

(ii) der Beseitigung von Redundanzfällen im Sprachsystems,

(iii) der funktionalen Ausweitung und Erleichterung der Funktionsweise der vorhandenen Sprachmittel sowie

(iv) Effizienz beim Ausbau des Sprachinventars gemeinsam ist. (vgl. Moser 1971: 93-101)

Als Beispiele für systemökonomische Spracherscheinungen nennt Moser u.a.:

(i) die Beseitigung der doppelten oder mehrfachen Kennzeichnung derselben Erscheinung (Wegfall von Doppelformen im Bereich der Orthographie, Lexik und Morphologie)

(ii) Neigung zu Kurzformen

(iii) Vereinfachung des Kasusgebrauchs

(iv) Gebrauch von verkürzenden Partizipialgruppen und partizipialen Fügungen anstelle des Relativsatzes 
(v) polyfunktionale Verwendung von Sprachzeichen (z.B. Präsens zur Bezeichnung der Zukunft)

(vi) Vereinfachung der Sprachformen (z.B. schwache Konjugation, Verzicht auf Nebensatz, Gebrauch von Nominalkonstruktionen)

(vii) Entlehnungen (Übernahme von „Fertigfabrikaten“)

(viii) Substantivierung

(ix) Bildung von Mehrwortkomposita bei Substantiven

(x) Neigung zu abstrakter Ausdrucksweise (z.B. durch Bildung von Oberbegriffen). (vgl. Moser 1971: 94-100)

In der Annahme, dass sich der Begriff der sprachlichen Ökonomie durch das Verhältnis zwischen sprachlichem Aufwand (Sprachsystem) und sprachlichem Ergebnis (Effizienz sprachlicher Kommunikation, erfolgreiche Vermittlung von Informationen) definiert, gleicht das system- und kulturgeschichtliche Konzept Mosers anderen Forschungsansätzen im Bereich kommunikativer Effizienz, wie beispielsweise dem Konzept der Easy Theorie von Martinet und Jespersen oder dem Modell des sprachlichen Wandels von Werner, wobei einzuräumen ist, dass in den meisten, vor allem synchronorientierten Sprachökonomietheorien die Minimierung des Aufwands besonders stark akzentuiert wird. ${ }^{1}$ Ähnliches meint auch Göpferich, wenn sie über Kürze als Prinzip fachsprachlicher Kommunikation spricht und auf lexikalische, syntaktische sowie nonverbale Mittel zur Kondensierung von Kommunikation verweist (vgl. Göpferich 2007).

\subsection{Informationsbezogene Ökonomie}

Während die systembezogene Ökonomie grundsätzlich den Aspekt des sprachlichen Aufwands und seiner Minimierung durch Ausdrucksverknappung, Formenvereinfachung, Polysemie oder Polyfunktionalität von Sprachzeichen in den Blick nimmt, bezweckt

\footnotetext{
${ }^{1}$ Ausführlicher zu Theorien sprachlicher Ökonomie u.a. bei Roelcke 2007: 726, insbesondere S. 10. Es wird u.a. auf Sprachstatistik, Terminologielehre, Minimalist Program oder Optimalitätstheorie verwiesen.
} 


\section{ComparativeLegilinguistics 29/2017}

die informationsbezogene Ökonomie die Erhöhung des Informationsgehalts und die Beschleunigung des Tempos der Übermittlung von Informationen sowie ihrer Aufnahme beim Empfänger u.a. durch die Vereinfachung der sprachlichen Gestaltung der Botschaft.

Im Idealfall ergäbe sich aus dem Verhältnis zwischen Systemund Informationsökonomie eine Kommunikationssituation, in der die Anwendung systemökonomischer Sprachlösungen sich als effektiv und zugleich effizient erweisen würde, d.h. mit wenigen Worten (geringem sprachlichem Aufwand) möglichst viele Informationen präzise und in einer für den Rezipienten verständlichen Weise (maximales Ergebnis) vermittelt werden, so dass im Endeffekt das kommunikative Ziel (Kommunikationseffizienz) erreicht wird. Hier knüpfen wir an die Unterscheidung zwischen Effektivität und Effizienz menschlicher Handlungen an, da sie sich auch für die Beschreibung des Spannungsverhältnisses zwischen Sprach- und Informationsökonomieals relevant erweist. Als effektiv wird eine Handlung dann bezeichnet, wenn sie ein bestimmtes Ergebnis erzielt. Die Aufwandsgröße spielt dabei keine Rolle. Auf die sprachliche Kommunikation bezogen wäre Effektivität erzielt, wenn eine Information vermittelt, vom Empfänger verstanden und die Kommunikationsabsicht des Senders erreicht wird. Erfolgt dies zugleich mit einem minimalen Aufwand (sprachökonomisch) und/oder mit einem maximalen Ergebnis (informationsökonomisch $\rightarrow$ Vermehrung der Informationsmenge + Schnelligkeit der Kommunikation), so wird die Kommunikation nicht nur effektiv, sondern auch effizient (vgl. Roelcke 2007: 14-15).Die sprachliche und kommunikative Effizienz muss allerdings nicht gleich dazu führen, dass die Kommunikation ineffektiv wird.

Wie Moser zu Recht bemerkt, erweist sich die Ausdrucksprägnanz unter dem Aspekt der Information nicht immer als effizienter, insbesondere dann, wenn die Schwerfälligkeit bzw. Unklarheit der Formulierung die schnelle Erfassung des Informationsgehalts verhindert, wie es bei vielen Mehrwortkomposita oder Kurzwörtern/Abkürzungen der Fall ist (vgl. Moser 1971: 108109).

Unter sprachlichen Erscheinungen, die informationsbezogen ökonomisch sind und den Textrezeptionsprozess fördern, nennt Moser u.a. den Verzicht auf Klammersatzkonstruktionen, die einen Überblick über den Satzinhalt als Ganzes erschweren und genauso wie 
analytische morphologische Formen das Informationstempo verlangsamen; die Hervorhebung der wichtigsten Mitteilung durch die Spitzenstellung im Satz, wodurch die Vermittlung von Hauptinformationen schneller erfolgen kann; die Nominalisierung als Mittel der Textverknappung (sie mindert die Zeichenanzahl) oder den Gebrauch von nominalen Umschreibungen, die eine inhaltliche Nuancierung ermöglichen (vgl. Moser 1971: 106-107).

Ergänzend kann hier noch in Anlehnung an Göpferich auf Kondensationsmittel auf lexikalischer, syntaktischer sowie textueller Ebene verwiesen werden wie die Verwendung und Bildung von Termini als „Textkondensate“ (Göpferich 2007: 414), der Gebrauch von Hypotaxe und Präsupposition oder der Einsatz nonverbaler Informationsträger bzw. der Formelschreibweise, die für einen komprimierten Stil sorgen (vgl. ebd.: 414-419).

\section{Sprach- und Informationsökonomie in einem Gesetzestext}

In Anbetracht der vorstehenden Überlegungen zur Auslegung und Weite des Begriffes sprachlicher Ökonomie sowie inhaltlicher Nuancierung ihrer Erscheinungsformen wollen wir uns nun der Analyse eines Gesetzestextes im Hinblick auf Mittel und Methoden zuwenden, die in dieser Textsorte die Textprägnanz und Informationsdichte und mithin die Sprach- und Informationsökonomie steigern.

Bei Vorschriftentexten haben Präzision, Prägnanz, Eindeutigkeit und Verständlichkeit der Texte besonderes Gewicht. Vorschriftentexte sollen sprachlich korrekt und grundsätzlich so verfasst sein, dass möglichst jedermann als Betroffener imstande ist, ihren Inhalt $\mathrm{zu}$ erschließen, damit er sein Verhalten entsprechend ausrichten kann. Da allerdings die Gesetzessprache als eine Fachsprache unmittelbar an das fachliche juristische Denken gebunden ist, erschließen sich dem Laien Begriffe und Aussagen in einem Gesetzestext nicht ohne weiteres, auch wenn der Text klar und allgemeinsprachlich verfasst ist. Zur Veranschaulichung reicht es darauf hinzuweisen, dass die Bedeutung vieler allgemeinsprachlicher 
Ausdrücke im juristischen Sprachgebrauch von ihrer Grundbedeutung abweicht und daher ohne entsprechendes Fachwissen missverstanden werden kann.

Für die Erreichung der Sprach- und Informationsökonomie in Gesetzestexten sorgt das Zusammenwirken von Kondensationsmitteln, die textübergreifend verwendet werden: Angefangen mit der pragmatischen Ebene, d.h. mit der Ausrichtung der Wortwahl und Textstruktur an den Geboten der Bestimmtheit und Knappheit; über die eigentliche Textstruktur, die eine überschaubare Gliederung in die kleinsten Einheiten aufweist, weiter über syntaktische Mittel, die für eine klare Gedankenfassung innerhalb der jeweiligen Grundeinheit sorgen; bis hin zur lexikalischen Ebene.

Eine klare und schematisierte Struktur eines Gesetzestextes erleichtert die Orientierung im Text. Hinzu kommen noch Titel und Überschriften (Zwischentitel), die zusätzliche Informationen zum Inhalt der einzelnen Vorschriften vermitteln und das Tempo der Informationsübermittlung dadurch beschleunigen, dass sie eine Information im Text leichter auffindbar machen. Wichtig für die Förderung der Textrezeption ist selbst die Grobeinteilung des Textes in einen allgemeinen und besonderen Teil, insbesondere im Hinblick auf die logische Verkettung von Begriffsbestimmungen, die im allgemeinen Teil legal definiert und anschließend im besonderen Teil auf konkrete Sachverhalte übertragen werden. Legaldefinitionen dienen auch der Sprachökonomie, da sie eine wiederholte Erklärung eines fachspezifischen Begriffes ersparen und die explizite Wiederaufnahme der Termini (Rekurrenz) ermöglichen.

Rezeptionsfördernd und sprachökonomisch wirkt des Weiteren die entsprechende Stilistik des Gesetzestextes. Den komprimierenden Stil von Vorschriftentexten machen v.a. die abstrakte Ausdrucksweise (Begriffsjurisprudenz) und das damit zusammenhängende Tendieren zum Gebrauch von Nominalisierung, Wortzusammensetzungen und Verweisungen aus.

Im Bereich der Syntax ist v.a. auf das Leitprinzip der Ausdruckprägnanz hinzuweisen. Sowohl in den deutschen als auch in den polnischen Empfehlungen für die sprachliche Gestaltung von Rechtsvorschriften wird ausdrücklich postuliert, dass ein Gedanke in möglichst einem Satz formuliert werden soll und dass innerhalb einer Grundeinheit jeweils eine Rechtsnorm zum Ausdruck gebracht 
wird. ${ }^{2}$ Dies soll grundsätzlich durch die Bildung von einfachen Sätzen erreicht werden. In der Tat kommt dies aber selten vor, da das Streben nach Informationsverdichtung zur Folge hat, dass in der Ausformulierung von Einzelvorschriften zusammengesetzte Sätze, oft Hypotaxen überwiegen, wodurch das Zusammenbringen mehrerer Normen in einer Vorschrift ermöglicht wird. Die Bildung einer Hypotaxe bedeutet allerdings nicht, dass der Inhalt unüberschaubar sein muss. Für Rezeptionsvereinfachung kann nämlich eine entsprechende Wortstellung im Satz sorgen, und zwar die Verschiebung von Kernaussagen an den Satzanfang bzw. in den Hauptsatz.

Um der Sprach- und Rezeptionsökonomie willen greift man auf lexikalischer Ebene meist zu Fachtermini, die einen Gesetzestext kondensieren, indem mit dem Gebrauch eines bestimmten Fachwortes gleich eine Bindung an das Abstrakte, das juristische Denken hergestellt wird. Es wird an das fachliche Vorwissen appelliert, das, falls vorhanden, die Texterschließung wesentlich vereinfacht. Eine Schlüsselfunktion kommt dabei meist den Nominalkonstrukten zu, da sie grundsätzlich für die Benennung von Sachverhalten zuständig sind und zugleich vom Standpunkt der Sprachökonomie als effizient einzustufen sind, da sie inhaltliche Verdichtung und Nuancierung mit sich bringen, u.a. durch die Vermehrung der Glieder bei Substantivkomposita, die Bildung der Relation der begrifflichen Überund Unterordnung oder die Bildung nominaler Umschreibungen. Ergänzend ist noch auf Attribuierung hinzuweisen, u.a. in Form partizipialer Fügungen, die allerdings wegen ihren Umfangs oft bei der Erfassung des Informationsgehalts als hinderlich empfunden werden können.

Nachdem nun eine Übersicht über die Hauptmittel sprachlicher Verknappung und textueller Kondensierung bei Gesetzestexten vermittelt worden ist, wollen wir nun an ausgewählten

\footnotetext{
${ }^{2}$ Vgl. Gesetzgebungsleitfaden. Handbuch der Rechtsförmlichkeit.
} Bundesministerium der Justiz und für Verbraucherschutz (Hrsg.), 2008.Unterhttp://hdr.bmj.de/page_b.1.index.html (Zugriffam 02.09.2016) undRozporządzenie Prezesa Rady Ministrów z dnia 20 czerwca 2002 r. w sprawie "Zasad techniki prawodawczej", unter http://isap.sejm.gov.pl/DetailsServlet?id=WDU20021000908 (Zugriffam 02.09.2016). 
Textbeispielen einige Verweisungstechniken genauer betrachten, um anschließend anhand der Fallanalyse die in der Einleitung gestellte Frage nach der Wechselwirkung zwischen Sprach- und Rezeptionsökonomie (Informationsökonomie)beantworten zu können.

\section{Verweisung als gesetzestechnisches Mittel der Textverknappung}

Verweise sind ein gesetzestechnisches Mittel, das die Steigerung der Wort- und Informationsökonomie auf textueller Ebene ermöglicht. Sie sind insbesondere nützlich, weil sie es erlauben, bereits andernorts dargelegte Inhalte nicht vollständig wiederholen $\mathrm{zu}$ müssen. Dies ermöglicht es, den Gesetzestext zu verknappen und dessen Inhalt auf das eigentlich Normative zu beschränken. Mit Verweisen wird auch die Konsistenz der Erlasstexte gewährleistet, da unbeabsichtigte Abweichungen in einzelnen Vorschriften vermieden werden und für vergleichbare Sachverhalte dieselben Tatbestandsvoraussetzungen sowie dieselben Rechtsfolgen angeführt werden (vgl. Höfler 2015: 326). Es wundert daher nicht, dass in Vorschriftentexten Verweise $\mathrm{zu}$ den meist verwendeten Kondensierungsmitteln gehören.

Falls sie richtig formuliert und nicht übermäßig eingesetzt werden, fördern Verweise mit Sicherheit die Textrezeption, da der Text durch die Vermeidung von Wiederholungen nicht nur dem Umfang nach knapper, sondern auch inhaltlich übersichtlicher wird. Die Bezugnahme auf andere Inhalte kann dabei unterschiedliche Formen annehmen. So kann sie über rein objektsprachliche Mittel erfolgen, d.h. durch sprachliche Rekurrenz auf einen bereits eingeführten Begriff, indem ein bereits definierter Terminus an einer anderen Textstelle wiederaufgenommen wird (vgl. Höfler 2015: 331).Hier ein Beispiel:

\section{Beispiel 1}

$\S 11$ Abs. I Nr. 5 StGB

„Im Sinne dieses Gesetzes ist (...) 
rechtswidrige Tat: nur eine solche, die den Tatbestand eines Strafgesetzes verwirklicht; (...).“(StGB 2013: 17)

$\S 12$ Abs. I StGB

„Verbrechen sind rechtswidrige Taten, die im Mindestmaß mit Freiheitsstrafe von einem Jahr oder darüber bedroht sind.“(dt. StGB 2013: 19)

Der Begriff der rechtswidrigen Tat wird zunächst legal definiert und anschließend nach dem geltenden Sprachgebrauch in einem anderen Paragraphen in Bezug auf den Begriff des Verbrechens (im Weiteren auch auf den Begriff des Vergehens) wieder verwendet. Durch die wörtliche Wiederholung des Ausdrucks wird das Rekurrenzverhältn is zwischen den beiden Begriffen transparent gemacht und damit Rezeptionseffizienz erzielt.

Eskann aber auch metasprachlich auf eine andere Textstelle verwiesen werden. Konstitutive (andersrestriktive)Verweisungen (der Bezugstext ist zugleich Teil der Ausgangsnorm) können dabei verschiedene Relationstypen zwischen Verweisungsobjekt und -norm bilden, z.B. den Verweis auf Tatbestandsvoraussetzungen, den Verweis auf die Rechtsfolgenseite anderer Rechtsnormen, die Beschreibung des Verhältnisses verschiedener Rechtsnormen zueinander, die Analogieverweisung, die Außenverweisung sowie inhaltsbezogene Verweisungen (vgl. Handbuch der Rechtsförmlichkeit, Teil B, Pkt. 4 Bezugnahme auf andere Texte, hier Abschnitte 219, 230 bis 235).

Explizite Verweise in Form einer metasprachlichen Wiederaufnahme beziehen sich also nicht auf bestimmte Gegenstände und Sachverhalte, sondern auf den Text als solchen. Solche Verweise erfordern einen kognitiven Mehraufwand, da sie den Textzusammenhang zerstören und durch ihre Metasprachlichkeit den Lesefluss beeinträchtigen. Tritt ein expliziter Verweis im Text auf, wird der Lese- und Verstehensprozess zeitweilig unterbrochen, die Aufmerksamkeit des Lesers weggelenkt, der dazu gezwungen wird, von der objektsprachlichen auf die metasprachliche Ebene zu wechseln.

Daher ist jeweils abzuwägen, ob eine Verweisung gegenüber einer Wiederholung tatsächlich eine Vereinfachung darstellt. Von grundsätzlicher Bedeutung sind dabei die Verständlichkeit eines 
Verweises, seine Präzision und Klarheit (vgl. Höfler 2015: 333-346). Diese sind dann daran zu messen, wie einfach es für den Leser ist, zu erkennen, worauf sich die Verweisung bezieht. Werden die Texttransparenz und -verständlichkeit nicht gewährleistet, so wird ein Verweis ineffektiv, da er den Rezeptionsprozess stört, anstatt ihn zu erleichtern. Hier einige Beispiele im Vergleich:

\title{
Beispiel 2
}

\section{$\S 44$ Abs. I S. 2 StGB}

„Ein Fahrverbot ist in der Regel anzuordnen, wenn in den Fällen einer Verurteilung nach § 315c Abs. I Nr. I Buchstabe a, Abs. 3 oder $\$ 316$ die Entziehung der Fahrerlaubnis nach § 69 unterbleibt.“(StGB 2013: 35)

Als eine der wortökonomischen Formulierungsweisen von Verweisen gilt die Formulierung mithilfe einer Präpositionalphrase mit der Präposition nach. Der Gebrauch des Funktionswortes nach kann sich allerdings trotz der erzielten Ausdrucksknappheit an Stellen als ineffizient erweisen, wo er zur Entstehung der syntaktischen Mehrbezüglichkeit führt. Es kann nämlich vorkommen, dass es mehr als ein Satzglied gibt, an das die Verweisung angebunden sein könnte. Diese Mehrdeutigkeit erschwert natürlich den Verstehensprozess. Der Leser wird vor die Notwendigkeit gestellt, unbedingt die Bezugsnorm zu konsultieren, um die Satzstruktur richtig erfassen zu können. Dies trifft auf den Verweis in Beispiel 2 zu. Unklar bleibt nämlich, ob sich der Verweis nach \& 69 auf die Nominalphrase die Entziehung der Fahrerlaubnis oder das Verb unterbleiben bezieht. Erst die Hinzuziehung der Bezugsnorm macht den Kontext eindeutig: Es geht um Fälle, in denen die Fahrerlaubnis entzogen wird. Wegen des Interpretationsproblems wird der Verweis aus informationsökonomischer Sicht offensichtlich ineffizient.

Auf eine Anbindungsmehrdeutigkeit stoßen wir auch in Beispiel 3.

\section{Beispiel 3}

\author{
$\S 46 b$ Abs. I Nr. 2 StGB \\ „Wenn der Täter einer Straftat (...) \\ freiwillig sein Wissen so rechtzeitig einer Dienststelle offenbart, dass eine \\ Tat nach $§$ 100a Abs. 2 der Strafprozessordnung, von deren Planung er
}


weiß, noch verhindert werden kann, kann das Gericht die Strafe nach § 49 Abs. I mildern (...).“(StGB 2013: 39)

Diesmal wird die Informationserfassung umso schwieriger, als wir es mit einem Außenverweis zu tun haben. Die Norm aus dem StGB wird explizit auf die Norm in der StPO bezogen. Dies beeinträchtigt das Tempo der Informationsübermittlung und macht den Zugriff auf einen anderen Gesetzestext erforderlich. Wieder lässt sich der Satzstruktur nicht eindeutig entnehmen, auf welchen Inhalt sich die Ausgangsnorm (nach $\S 100 a$ Abs. 2 StPO) bezieht, auf das Nomen Tat oder die Verbalphrase verhindert werden kann. Schlägt man in der StPO nach, so zeigt sich, dass die Norm auf den in der Bezugsnorm verzeichneten Katalog schwerer Straftaten verweist.

Die beiden Beispiele zeigen, dass die Forderungen nach Ausdrucksknappheit und Präzision manchmal in Konflikt zueinander geraten können. Die Formulierung des Verweises mittels der Präpositionalphrase macht ihn zwar kompakter, aber weniger präzise und beeinträchtigt dadurch das Verständnis. Anstatt die Präpositionalphrase mit der Präposition nach zu gebrauchen, könnte man in den genannten Fällen den Verweis als Partizipialkonstruktion (z.B. eine in \$ $100 a$ Abs. 2 der Strafprozessordnung genannte Tat) formulieren, die zwar etwas länger, dafür aber eindeutig und somit rezeptionsfördernd ist.

\title{
Beispiel 4
}

\author{
$\S 53$ Abs. 4 StGB \\ „§52 Abs. 3 und 4 Satz 2 gilt sinngemäß.“(StGB 2013: 45) \\ $\S 130$ a Abs. 3 StGB \\ „§ 86 Abs. 3 gilt entsprechend.“ (StGB 2013: 177)
}

Beispiel 4 beinhaltet zwei weitere Formen der Verweise, und zwar die sog. nackte Verweisung (vgl. Müller 1968: 191; Höffler 2015: 342) und die Analogieverweisung. Im Hinblick auf Informations- und Rezeptionsökonomie sind nackte Verweisungen grundsätzlich zu vermeiden, da sie für sich allein genommen nicht erkennen lassen, welcher Gegenstand oder Sachverhalt hier gemeint ist. Wie Müller betont, wirkt das einfache Zitieren von Vorschriften ohne irgendwelche Kontextannäherung auf die Leser „orakelhaft“ (Müller 1968: 191). Um sich eine Vorstellung davon zu machen, was 


\title{
ComparativeLegilinguistics 29/2017
}

an der Textstelle überhaupt geregelt wird, ist der Leser gezwungen, die Bezugsnorm zu lesen. Bei Analogieverweisungen dagegen wird der Bezugstext nicht wörtlich, sondern nur sinngemäß übernommen. Hervorgehoben wird diese Textrelation durch obligatorisches Hinzufügen der Adverbialbestimmung sinngemäß oder entsprechend. Die Formulierung über die sinngemäße/entsprechende Geltung bzw. Anwendung von Vorschriften ist für den Leser, insbesondere wenn er nicht über juristische Fachkenntnisse verfügt, nicht ohne weiteres nachvollziehbar. Oft entstehen daher Unklarheiten. Dies ist darauf zurückzuführen, dass der Gebrauch dieser Formulierung das Risiko der Interpretationsvielfalt mit sich bringt (vgl. Wronkowska / Zieliński 2004: 300). Sinngemäß/entsprechend kann sich auf die Anwendung der Bezugsnorm mit Abwandlungen, ohne Abwandlungen oder die Nichtanwendung der Bezugsnorm auf die Ausgangsnorm beziehen. Um den Regelungsinhalt der Ausgangsnorm und die Analogie zwischen der Ausgangs- und Bezugsnorm verständlicher zu machen, können Abwandlungen ausdrücklich angegeben werden. Dies ist aber in den beiden Vorschriften aus Beispiel 4 nicht der Fall, wodurch die Textrezeption insofern erschwert und verlangsamt wird, als der Leser eine Inhaltsanalyse vornehmen muss, um zu erschließen, in welchem Umfang und auf welche Sachverhalte die Bezugsnorm unverändert oder verändert Anwendung findet. Durch die Anwendung von nackten Verweisungen wird die Textrezeption noch stärker behindert. Dem könnte man aber durch die Umschreibung des Gegenstands der Bezugsnorm in der Ausgangsnorm entgegenwirken. Eine solche inhaltliche Ergänzung wäre aus wortökonomischer Sicht zwar weniger effizient, dafür aber rezeptionsfördernd, da sie die Funktion der Verweisungsnorm innerhalb des Regelungskomplexes, in dem sie erscheint, überschaubarer macht.

\section{Beispiel 5}

\author{
$\S 265 \mathrm{a}$ Abs. $3 \mathrm{StGB}$ \\ „Die $§ 247$ und 248a gelten entsprechend.“(StGB 2013: 291)$$
\downarrow
$$ \\ $\S 248 \mathrm{a} \mathrm{StGB}$ \\ „Der Diebstahl und die Unterschlagung geringwertiger Sachen werden in \\ den Fällen der $\$ \S 242$ und 246 nur auf Antrag verfolgt, es sei denn, dass die \\ Strafverfolgungsbehörde wegen des besonderen öffentlichen Interesses an
}


der Strafverfolgung ein Einschreiten von Amts wegen für geboten hält.“ (StGB 2013: 273)

Die in Beispiel 5 eingesetzte Binnen- und zum Teil Analogieverweisungen dienen der Beschreibung des Verhältnisses verschiedener Rechtsnormen zueinander. In $\S 265 \mathrm{a}$ werden die Tatbestandsmerkmale des Erschleichens von Dienstleistungen beschrieben. Abs. 3 des Paragraphen verweist auf ein Analogieverhältnis zwischen der Ausgangsnorm und zwei Bezugsnormen. § 247 besagt, wenn ,durch einen Diebstahl oder eine Unterschlagung ein Angehöriger, der Vormund oder der Betreuer verletzt [ist] oder der Verletzte mit dem Täter in häuslicher Gemeinschaft [lebt], so wird die Tat nur auf Antrag verfolgt" (StGB 2013: 271). Die Verfolgung auf Antrag thematisiert auch $\S 248 \mathrm{a}$, in dem allerdings auf den Umstand des Diebstahls und der Unterschlagung geringwertiger Sachen hingewiesen sowie auf zwei weitere Vorschriften verwiesen wird, deren Regelungsgegenstand entsprechend die Entwendung einer fremden beweglichen Sache zwecks rechtswidriger Zueignung und rechtswidrige Zueignung selbst darstellen. Es wird somit eine Verweisungskette gebildet, die die logischen Zusammenhänge innerhalb des Regelungskomplexes wenig, wenn überhaupt, überschaubar macht. Da die jeweilige Bezugsnorm ihrerseits Verweisungen enthält, müssen neben Ausgangs- und Bezugsnorm weitere Vorschriften mitgelesen werden, um zu ermitteln, was eigentlich geregelt wird. Neben den bereits angesprochenen interpretationsbedingten Verständnisproblemen bei Analogieverweisungen sorgt auch die logische Verkettung von Oberund Unterbegriffen (Betrug, Untreue, Diebstahl und Unterschlagung), unter welche das Erschleichen von Dienstleistungen kategorisiert wird, für eine weitere Rezeptionsstörung. Die wortökonomische Verweisungstechnik erweist sich in diesem Falle als äußerst ineffizient, da der kognitive und zeitliche Aufwand, der vonnöten ist, um den Regelungsinhalt komplex erfassen zu können, unvergleichbar höher ist, als wenn in der Ausgangsnorm die einzelnen Bezugsnormen explizite wiederaufgenommen würden. 


\section{Schlussbetrachtung}

Ein Vorschriftentext befindet sich in einem gewissen Spannungsfeld zwischen dem Knappheits- und Bestimmtheitsgebot. Einerseits soll er möglichst knapp und ohne Redundanz formuliert sein, damit er seine pragmatische und kommunikative Funktion erfüllen kann. Andererseits muss er präzise und eindeutig sein, damit seine restriktive Funktion gesichert wird und keine so weit gehenden Interpretationszweifel entstehen, die die Textrezeption unmöglich machen. Die analysierten Beispiele zeigten, dass die genannten Forderungen manchmal in Konflikt zueinander geraten können.

Verweisungen werden in Gesetzestexten sehr häufig, sogar übermäßig gebraucht. Dies lässt sich v.a. auf ihren wortökonomischen Charakter, aber nicht selten auch auf die Bequemlichkeit der Gesetzesredaktoren zurückführen. Ob Verweise im Hinblick auf den Lese- und Verstehensprozess effizient und nicht nur effektiv werden, hängt von der Präzision ihrer Formulierung ab. Es zeigte sich, dass in manchen Fällen eine längere Formulierung eindeutiger wäre und umso effizienter, als der Leser nicht gezwungen wird, zuvor die Bezugsnorm zu konsultieren, oder die Textstelle mehrmals zu lesen, um den inhaltlichen Zusammenhang erschließen zu können.

Die Analyse hat also gezeigt, dass Sprachökonomie nicht immer Rezeptionsförderung bedeutet. Einen Beweis dafür konnten insbesondere Beispiele für Verweisungen liefern, in denen das Nebeneinander von mehreren Verweisungstechniken (z.B. der Analogie-, Binnen- und Kettenverweisung) wegen der inhaltlichen Unübersichtlichkeit zu gravierenden Rezeptionssproblemen führte.

Zum Schluss muss gesagt werden, dass der ideale Grad der Prägnanz mit Sicherheit a priori nicht bestimmbar ist, und es kommt allein dem Textproduzenten, dem Gesetzesredaktor zu, zu entscheiden, welche Lösung in der jeweiligen Situation effizienter ist und ob er in einem bestimmten Kommunikationsrahmen der sprachlichen Kürze oder der Rezeptionsökonomie den Vorzug gibt. 
Karolina Kęsicka: ZwischenSprach- und ...

\section{Bibliographie}

Deutsches Strafgesetzbuch vom 17. Oktober 1964 zuletzt geändert 21.1.2013 (BGB1. I S. 95). Übersetzung ins Polnische. Übersetzt von Ewa Schwierskott-Matheson. Regensburg: De Iure 2013 (bilingual Polnisch-Deutsch).

Gesetzgebungsleitfaden. Handbuch der Rechtsförmlichkeit. 2008. Bundesministerium der Justiz und für Verbraucherschutz (Hrsg.). http://hdr.bmj.de/page_b.1.index.html (Zugriff am 02.09.2016).

Göpferich, Susanne. 2007. Kürze als Prinzip fachsprachlicher Kommunikation. In Sprachliche Kürze. Konzeptuelle, strukturelle und pragmatische Aspekte, hrsg. von Jochen A. Bär, Thorsten Roelcke und Anja Steinhauer, 412-434.Berlin: de Gruyter.

Höfler, Stefan. 2015. Die Redaktion von Verweisen unter dem Aspekt der Verständlichkeit. Leges 2: 325-349.

Martinet, André. 1960. Eléments de linguistique générale. Paris: Armand Colin.

Moser, Hugo. 1971. Typen sprachlicher Ökonomie im heutigen Deutsch. In Sprache und Gesellschaft. Beiträge zur soziolinguistischen Beschreibung der deutschen Gegenwartssprache, hrsg. von Hugo Moser, 89-117. Düsseldorf: Pädagogischer Verlag Schwann.

Müller, Hanswerner. 1968. Handbuch der Gesetzgebungstechnik. Köln: Heymanns.

Roelcke, Thorsten. 2007. Effizienz sprachlicher Kommunikation. In Sprachliche Kürze. Konzeptuelle, strukturelle und pragmatische Aspekte, hrsg. von Jochen A. Bär, Thorsten Roelcke und Anja Steinhauer, 7-26. Berlin: de Gruyter.

Wronkowska, Sławomira und Maciej Zieliński. 2004. Komentarz do zasad techniki prawodawczej $\mathrm{z}$ dnia 20 czerwca $2002 \mathrm{r}$. Warszawa: Wydawnictwo Sejmowe. 